\title{
The construction and deformation of BiHom-Novikov agebras
}

\author{
Shuangjian Guo ${ }^{1}$, Xiaohui Zhang ${ }^{2}$, Shengxiang Wang ${ }^{3 *}$ \\ 1. School of Mathematics and Statistics, Guizhou University of Finance and Economics \\ Guiyang 550025, P. R. of China \\ 2. School of Mathematical Sciences, Qufu Normal University \\ Qufu 273165, P. R. of China \\ 3. School of Mathematics and Finance, Chuzhou University \\ Chuzhou 239000, P. R. of China
}

\begin{abstract}
BiHom-Novikov agebra is a generalized Hom-Novikov algebra endowed with two commuting multiplicative linear maps. The main purpose of this paper is to show that two classes of BiHom-Novikov algebras can be constructed from BiHom-commutative algebras together with derivations and BiHom-Novikov algebras with Rota-Baxter operators, respectively. We show that quadratic BiHom-Novikov algebras are associative algebras and the sub-adjacent BiHomLie algebras of BiHom-Novikov algebras are 2-step nilpotent. Moreover, we develop the 1-parameter formal deformation theory of BiHom-Novikov algebras
\end{abstract}

Key words: BiHom-Novikov agebra, BiHom-Lie algebra, BiHom-associative algebra, derivation, Rota-Baxter operator

2010 Mathematics Subject Classification: 17B75, 17B40, 17B55

\section{INTRODUCTION}

Novikov algebras were introduced in connection with the Poisson brackets of hydrodynamic type [4, 10] and Hamiltonian operators in the formal variational calculus [12, 30]. The theoretical study of Novikov algebras was started by Zelmanov and Tikhov [34] and Filipov [11]. But the term Novikov algebra was first used by Osborn [27]. The left multiplication operators of a Novikov algebra form a Lie algebra. Thus, it is effective to relate the study of Novikov algebras to the theory of Lie algebras [6]. Novikov algebras

${ }^{*}$ Corresponding author(Shengxiang Wang): wangsx-math@163.com 
are a special class of left-symmetric algebras (or under other names such as pre-Lie algebras, quasi-associative algebras and Vinberg algebras), arising from the study of affine manifolds, affine structures and convex homogeneous cones [3]. Left-symmetric algebras have close relations with many important fields in mathematics and mathematical physics, such as infinite-dimensional Lie algebras [4], classical and quantum Yang-Baxter equation, quantum field theory and so on.

A quadratic Novikov algebra, introduced in [7], is a Novikov algebra with a symmetric nondegenerate invariant bilinear form. The motivation for studying quadratic Novikov algebras came from the fact that Lie algebras or associative algebras with symmetric nondegenerate invariant bilinear forms have important applications in several areas of mathematics and physics, such as the structure theory of finite-dimensional semisimple Lie algebras, the theory of complete integrable Hamiltonian systems and the classification of statistical models over two-dimensional graphs.

The deformation is a tool to study a mathematical object by deforming it into a family of the same kind of objects depending on a certain parameter. The deformation theory was introduced by Gerstenhaber for rings and algebras [13]-[16], by Kubo and Taniguchi for Lie triple systems [21], by Ma, Chen and Lin for Hom-Lie Yamaguti algebras [23] and Hom-Novikov superalgebras [28, by Bai and Meng for Novikov algebras [5]. They studied 1- parameter formal deformations and established the connection between the cohomology groups and infinitesimal deformations.

As generalizations of Lie algebras, Hom-Lie algebras were introduced motivated by applications to physics and to deformations of Lie algebras, especially Lie algebras of vector fields. The notion of Hom-Lie algebras was firstly introduced by Hartwig, Larsson and Silvestrov to describe the structure of certain $q$-deformations of the Witt and the Virasoro algebras, see [2, 8, 19, 20]. More precisely, a Hom-Lie algebras are different from Lie algebras as the Jacobi identity is replaced by a twisted form using a morphism.

The twisting of parts of the defining identities was transferred to other algebraic structures. In [24, 25, 26, Makhlouf and Silvestrov introduced the notions of Hom-associative algebras, Hom-coassociative coalgebras, Hom-bialgebras and Hom-Hopf algebras. The original definition of a Hom-bialgebra involved two linear maps, one twisting the associativity condition and the other one twisting the coassociativity condition.

Yau in 32 introduced Hom-Novikov algebras, in which the two defining identities are twisted by a linear map. It turned out that Hom-Novikov algebras can be constructed from Novikov algebras, commutative Hom-associative algebras and Hom-Lie algebras along with some suitable linear maps. And Yuan in [31] introduced quadratic Hom- Novikov algebras. Later, Later, Zhang, Hou and Bai in 35] defined a Hom-Novikov superalgebra as a twisted generalization of Novikov superalgebras.

A Bihom-algebra is an algebra in such a way that the identities defining the structure 
are twisted by two homomorphisms $\alpha, \beta$. This class of algebras was introduced from a categorical approach in [18] as an extension of the class of Hom-algebras. When the two linear maps are same automorphisms, Bihom-algebras will be return to Hom-algebras. These algebraic structures include Bihom-associative algebras, Bihom-Lie algebras and Bihom-bialgebras. The representation theory of Bihom-Lie algebras was introduced by Cheng and Qi in [9], in which, Bihom-cochain complexes, derivation, central extension, derivation extension, trivial representation and adjoint representation of Bihom-Lie algebras were studied. More applications of BiHom-algebras, BiHom-Lie superalgebras and BiHom-Lie colour algebras can be found in ([1], 22, 229]).

In the present paper, we consider the construction and deformation of BiHom-Novikov agebras.

In Section 2, we focus on BiHom-Novikov algebras. We establish the relationships between BiHom- Novikov algebras and BiHom-Lie algebras as well as the relations between BiHom-Novikov algebras and Hom-Novikov algebras. Also, we provide a construction of BiHom-Novikov algebras from a commutative associative algebra together with derivations and BiHom-Novikov algebras with Rota-Baxter operators, respectively.

In Section 3, we introduce the notion of quadratic BiHom-Novikov algebras and the relationship between BiHom-Novikov algebras and sub-adjacent BiHom-Lie algebras. We also show quadratic BiHom- Novikov algebras are associative algebras and the sub-adjacent BiHom-Lie algebras of BiHom-Novikov algebras are 2-step nilpotent.

In Section 4, we define low orders coboundary operator and give low order cohomology groups of BiHom-Novikov algebras. We show that the cohomology group is suitable for this 1-parameter formal deformation theory.

\section{Preliminaries}

Throughout the paper, all algebraic systems are supposed to be over a field $\mathbb{K}$. Any unexplained definitions and notations can be found in [18] and 33.

In this section we recall some basic definitions and results related to our paper.

1.1. BiHom-associative algebra $\mathrm{A}$ BiHom-associative algebra is a 4-tuple $(A, \mu, \alpha, \beta)$, where $A$ is a $k$-linear space, $\alpha: A \rightarrow A, \beta: A \rightarrow A$ and $\mu: A \otimes A \rightarrow A$ are linear maps, with notation $\mu(a \otimes b)=a b$, satisfying the following conditions, for all $a, a^{\prime}, a^{\prime \prime} \in A$ :

$$
\begin{aligned}
& \alpha \circ \beta=\beta \circ \alpha, \\
& \alpha\left(a a^{\prime}\right)=\alpha(a) \alpha\left(a^{\prime}\right), \beta\left(a a^{\prime}\right)=\beta(a) \beta\left(a^{\prime}\right), \\
& \alpha(a)\left(a^{\prime} a^{\prime \prime}\right)=\left(a a^{\prime}\right) \beta\left(a^{\prime \prime}\right) .
\end{aligned}
$$

And the maps $\alpha, \beta$ are called the structure maps of $A$. 
Clearly, a Hom-associative algebra $(A, \mu, \alpha)$ can be regarded as the BiHom-associative algebra $(A, \mu, \alpha, \alpha)$.

1.2. BiHom-Lie algebra A BiHom-Lie algebra is a 4 -tuple $(L,[\cdot, \cdot], \alpha, \beta)$, where $L$ is a $k$-linear space, $\alpha: L \rightarrow L, \beta: L \rightarrow L$ and $[\cdot, \cdot]: L \otimes L \rightarrow L$ are linear maps, satisfying the following conditions, for all $a, a^{\prime}, a^{\prime \prime} \in A$ :

$$
\begin{aligned}
& \alpha \circ \beta=\beta \circ \alpha \\
& \alpha\left[a, a^{\prime}\right]=\left[\alpha(a), \alpha\left(a^{\prime}\right)\right], \beta\left[a, a^{\prime}\right]=\left[\beta(a), \beta\left(a^{\prime}\right)\right], \\
& {\left[\beta(a), \alpha\left(a^{\prime}\right)\right]=-\left[\beta\left(a^{\prime}\right), \alpha(a)\right] .} \\
& {\left[\beta^{2}(a),\left[\beta\left(a^{\prime}\right), \alpha\left(a^{\prime \prime}\right)\right]\right]+\left[\beta^{2}\left(a^{\prime}\right),\left[\beta\left(a^{\prime \prime}\right), \alpha(a)\right]\right]+\left[\beta^{2}\left(a^{\prime \prime}\right),\left[\beta(a), \alpha\left(a^{\prime}\right)\right]\right]=0 .}
\end{aligned}
$$

Obviously, a Hom-Lie algebra $(L,[\cdot, \cdot], \alpha)$ is a particular case of a BiHom-Lie algebra, namely $(L,[\cdot, \cdot], \alpha, \alpha)$. Conversely, a BiHom-Lie algebra $(L,[\cdot, \cdot], \alpha, \alpha)$ with bijective $\alpha$ is the Hom-Lie algebra $(L,[\cdot, \cdot], \alpha)$.

1.3. Hom-Novikov algebra A Hom-Novikov algebra is a triple $(A, \mu, \alpha)$ consisting of a vector space $A, \alpha: A \rightarrow A$ is a algebra homomorphisms, $\mu: A \otimes A \rightarrow A$ is an even bilinear map, with notation $\mu(a \otimes b)=a b$ satisfying

$$
\begin{aligned}
& (x y) \alpha(z)=(x z) \alpha(y), \\
& (x y) \alpha(z)-\alpha(x)(y z)=(y x) \alpha(z)-\alpha(y)(x z),
\end{aligned}
$$

for all $x, y, z \in A$.

\section{BiHom-Novikov algebras}

In this section, we establish the relationships between BiHom-Novikov algebras and Hom-Novikov algebras as well as the relations between BiHom-Novikov algebras and BiHom-Lie algebras. Also, we provide a construction of BiHom-Novikov algebras from a commutative BiHom-associative algebra along with a suitable linear map but not a derivation.

Definition 2.1. A BiHom-Novikov algebra is a 4-tuple $(A, \mu, \alpha, \beta)$ consisting of a vector space $A, \alpha: A \rightarrow A$ and $\beta: A \rightarrow A$ are algebras homomorphisms, $\mu: A \otimes A \rightarrow A$ is an even bilinear map, with notation $\mu(a \otimes b)=a b$ satisfying

$$
\begin{aligned}
& \alpha \circ \beta=\beta \circ \alpha, \\
& (x y) \alpha(z)=(x z) \alpha(y), \\
& (\beta(x) \alpha(y)) \beta(z)-\alpha \beta(x)(\alpha(y) z)=(\beta(y) \alpha(x)) \beta(z)-\alpha \beta(y)(\alpha(x) z),
\end{aligned}
$$

for all $x, y, z \in A$. 
Clearly, Hom-Novikov algebras and Novikov algebras are examples of BiHom-Novikov algebras by setting $\beta=\alpha$ and $\alpha=\beta=i d$, where $i d$ is the identity map. It was shown in [33] that any Novikov algebra can be deformed into a Hom-Novikov algebra along with an algebra endomorphism.

Proposition 2.2. Let $(A, \mu, \alpha, \beta)$ be a BiHom-Novikov algebra, for all $x, y, z \in A$, we have

$$
\begin{gathered}
{[\beta(x), \alpha(y)] \alpha^{2}(z)+[\beta(y), \alpha(z)] \alpha^{2}(x)+[\beta(z), \alpha(x)] \alpha^{2}(y)=0,} \\
\beta^{2}(z)[\beta(x), \alpha(y)]+\beta^{2}(x)[\beta(y), \alpha(z)]+\beta^{2}(y)[\beta(z), \alpha(x)]=0,
\end{gathered}
$$

where $\mu(x, y)=x y$ and $[\beta(x), \alpha(y)]=\beta(x) \alpha(y)-\beta(x) \alpha(y)$.

Proof. For any $x, y, z \in A$, we have

$$
\begin{aligned}
& {[\beta(x), \alpha(y)] \alpha^{2}(z)=(\beta(x) \alpha(y)) \alpha^{2}(z)-(\beta(y) \alpha(x)) \alpha^{2}(z),} \\
& {[\beta(y), \alpha(z)] \alpha^{2}(x)=(\beta(y) \alpha(z)) \alpha^{2}(x)-(\beta(z) \alpha(y)) \alpha^{2}(x),} \\
& {[\beta(z), \alpha(x)] \alpha^{2}(y)=(\beta(z) \alpha(x)) \alpha^{2}(y)-(\beta(x) \alpha(z)) \alpha^{2}(y),}
\end{aligned}
$$

Since equation (2.2) holds for all $x, y, z \in A$, we have

$$
\begin{aligned}
& {[\beta(x), \alpha(y)] \alpha^{2}(z)+[\beta(y), \alpha(z)] \alpha^{2}(x)+[\beta(z), \alpha(x)] \alpha^{2}(y) } \\
= & (\beta(x) \alpha(y)) \alpha^{2}(z)-(\beta(y) \alpha(x)) \alpha^{2}(z)+(\beta(y) \alpha(z)) \alpha^{2}(x)-(\beta(z) \alpha(y)) \alpha^{2}(x) \\
& +(\beta(z) \alpha(x)) \alpha^{2}(y)-(\beta(x) \alpha(z)) \alpha^{2}(y) \\
= & 0,
\end{aligned}
$$

which proves equation (2.4). Now using equations (2.2) and (2.4), we have

$$
\begin{aligned}
& \beta^{2}(z)[\beta(x), \alpha(y)]+\beta^{2}(x)[\beta(y), \alpha(z)]+\beta^{2}(y)[\beta(z), \alpha(x)] \\
= & \beta^{2}(z)(\beta(x) \alpha(y))+\beta^{2}(x)(\beta(y) \alpha(z))-\beta^{2}(x)(\beta(z) \alpha(y)) \\
& +\beta^{2}(y)(\beta(z) \alpha(x))-\beta^{2}(y)(\beta(x) \alpha(z)) \\
= & \beta^{2}(z)(\beta(x) \alpha(y))-\beta^{2}(x)(\beta(z) \alpha(y))+\beta^{2}(x)(\beta(y) \alpha(z))-\beta^{2}(y)(\beta(x) \alpha(z)) \\
& \beta^{2}(y)(\beta(z) \alpha(x))-\beta^{2}(z)(\beta(y) \alpha(x)) \\
= & \left(\alpha^{-1} \beta^{2}(z) \beta(x)-\alpha^{-1} \beta^{2}(x) \beta(z)\right) \alpha \beta(y)+\left(\alpha^{-1} \beta^{2}(x) \beta(y)-\alpha^{-1} \beta^{2}(y) \beta(x)\right) \alpha \beta(z) \\
& \left(\alpha^{-1} \beta^{2}(x) \beta(y)-\alpha^{-1} \beta^{2}(y) \beta(x)\right) \alpha \beta(z) \\
= & {\left[\alpha^{-1} \beta^{2}(z), \beta(x)\right] \alpha \beta(y)+\left[\alpha^{-1} \beta^{2}(y), \beta(z)\right] \alpha \beta(x)+\left[\alpha^{-1} \beta^{2}(x), \beta(y)\right] \alpha \beta(z) } \\
= & \alpha^{-1} \beta\left([\beta(x), \alpha(y)] \alpha^{2}(z)+[\beta(y), \alpha(z)] \alpha^{2}(x)+[\beta(z), \alpha(x)] \alpha^{2}(y)\right) \\
= & 0 .
\end{aligned}
$$

And this finishes the proof. 
Corollary 2.3. Let $(A, \mu, \alpha, \beta)$ be a BiHom-Novikov algebra, Define a bilinear map $[\cdot, \cdot]: A \times A \rightarrow A$ by

$$
[x, y]=\mu(x \otimes y)-\mu\left(\alpha^{-1} \beta(y) \otimes \alpha \beta^{-1} x\right), \text { for any } x, y \in A .
$$

Then $(A,[\cdot, \cdot], \alpha, \beta)$ is a BiHom-Lie algerba.

Definition 2.4. Let $(A, \mu, \alpha, \beta)$ be a BiHom-Novikov algebra, Which is called

(i) regular if $\alpha, \beta$ are algebra automorphism.

(ii) involutive if $\alpha, \beta$ are involution, i.e., $\alpha^{2}=\beta^{2}=i d$.

Yau [32] and Yuan [31] gave a way to construct Hom-Novikov algebras, starting from a Novikov algebra and an algebra endomorphism. In the following, we provide a construction of BiHom-Novikov algebras from Hom-Novikov algebras and Novikov algebras along with algebra automorphisms.

Proposition 2.5. Let $(A, \mu, \alpha, \beta)$ be an involutive BiHom-Novikov algebra, define a new multiplication on $A$ by $x * y=\alpha(x) \beta(y)$. Then $(A, *)$ is a Novikov algebra.

Proof. We write $\mu(x \otimes y)=x y$ and $x * y=\beta(x) \alpha(y)$, for all $x, y \in A$. Hence, it needs to show

$$
\begin{array}{r}
(x * y) * z=(x * z) * y \\
(x * y) * z-x *(y * z)=(y * x) * z-y *(x * z),
\end{array}
$$

for all $x, y, z \in A$. Since $\alpha, \beta$ are involution, we have

$$
(x * y) * z=\alpha(\alpha(x) \beta(y)) \beta(z)=\left(\alpha^{2}(x) \alpha \beta(y)\right) \beta(z)=
$$

Similar, we have $(x * z) * y=\alpha(\alpha(x) \beta(z)) \beta(y)=\left(\alpha^{2}(x) \alpha \beta(z)\right) \beta(y)$, from which equation (2.6) follows since $(A, \mu, \alpha, \beta)$ is an involutive BiHom-Novikov algebra. Furthermore, using equation $(2.3)$, we have

$$
\begin{aligned}
& (x * y) * z-x *(y * z) \\
= & \alpha(\alpha(x) \epsilon(y)) \beta(z)-\alpha(x) \beta(\alpha(y) \beta(z)) \\
= & \left(\alpha^{2}(x) \alpha \beta(y)\right) \beta(z)-\alpha(x)\left(\alpha \beta(y) \beta^{2}(z)\right) \\
= & \left(\beta^{2}(y) \alpha \beta^{-1}(x)\right) \beta(z)-\alpha(y)(\alpha \beta(x) z) \\
= & \alpha(\alpha(y) \beta(x)) \beta(z)-\alpha(y) \beta(\alpha(x) \beta(z)) \\
= & (y * x) * z-y *(x * z),
\end{aligned}
$$

which proves equation (2.7) and the proposition.

Proposition 2.6. Let $(A, \mu)$ be a Novikov algebra, and $\alpha, \beta: A \rightarrow A$ are linear maps satisfying $\alpha \circ \beta=\beta \circ \alpha, \alpha(x \otimes y)=\alpha(x) \alpha(y)$ and $\beta(x y))=\beta(x) \beta(y)$, for all $x, y \in A$, and we define a new multiplication on $A$ by $x \star y=\alpha(x) \beta(y)$. Then $(A, \star, \alpha, \beta)$ is a BiHom-Novikov algebra. 
Proof. We only prove (2.3) and leave the rest to the reader: for any $x, y, z \in A$, we have

$$
\begin{aligned}
& (\beta(x) \star \alpha(y)) \star \beta(z)-\alpha \beta(x) \star(\alpha(y) \star z) \\
= & \alpha(\alpha \beta(x) \alpha \beta(y)) \beta^{2}(z)-\alpha^{2} \beta(x)\left(\alpha^{2} \beta(y) \beta^{2}(z)\right) \\
= & \left(\alpha^{2} \beta(y) \alpha^{2} \beta(x)\right) \beta^{2}(z)-\alpha^{2} \beta(y)\left(\alpha^{2} \beta(x) \beta^{2}(z)\right) \\
= & \alpha(\alpha \beta(y) \alpha \beta(x)) \beta^{2}(z)-\alpha^{2} \beta(y)\left(\alpha^{2} \beta(x) \beta^{2}(z)\right) \\
= & (\beta(y) \star \alpha(x)) \star \beta(z)-\alpha \beta(y) \star(\alpha(x) \star z)
\end{aligned}
$$

Proposition 2.7. Let $(A, \mu, \alpha, \beta)$ be a regular BiHom-Novikov algebra, define a new multiplication on $A$ by $[x, y]^{\prime}=\left[\alpha^{-1}(x), \beta^{-1}(y)\right]$ where $[x, y]=x y-\alpha^{-1}(\beta(y)) \alpha\left(\beta^{-1}(x)\right)$, for all $x, y \in A$, then $\left(A,[\cdot, \cdot]^{\prime}\right)$ is a Lie algebra. In particular, if $\alpha, \beta$ are involution and we define multiplication on $A$ by $[x, y]^{\prime \prime}=[\alpha(x), \beta(y)]$, then $\left(A,[\cdot, \cdot]^{\prime \prime}\right)$ is a Lie algebra.

Proof. For any $x, y, z \in A$, we have

$$
\begin{aligned}
& {\left[[x, y]^{\prime}, z\right]^{\prime} } \\
= & {\left[\left[\alpha^{-2}(x), \alpha^{-1} \beta^{-1}(y)\right], \beta^{-1}(z)\right] } \\
= & {\left[\alpha^{-2}(x), \alpha^{-1} \beta^{-1}(y)\right] \beta^{-1}(z)-\alpha^{-1}(z) \alpha \beta^{-1}\left[\alpha^{-2}(x), \alpha^{-1} \beta^{-1}(y)\right] } \\
= & \alpha^{-2} \beta^{-1}\left([\beta(x), \alpha(y)] \alpha^{2}(z)-\alpha \beta(z)\left[\alpha(x), \alpha^{2} \beta^{-1}(y)\right]\right)
\end{aligned}
$$

Similarly, we have

$$
\begin{aligned}
& {\left[[y, z]^{\prime}, x\right]^{\prime}=\alpha^{-2} \beta^{-1}\left([\beta(y), \alpha(z)] \alpha^{2}(x)-\alpha \beta(x)\left[\alpha(y), \alpha^{2} \beta^{-1}(z)\right]\right),} \\
& {\left[[z, x]^{\prime}, y\right]^{\prime}=\alpha^{-2} \beta^{-1}\left([\beta(z), \alpha(x)] \alpha^{2}(y)-\alpha \beta(y)\left[\alpha(z), \alpha^{2} \beta^{-1}(x)\right]\right),}
\end{aligned}
$$

Then it follows from equations (2.4) and (2.5) that

$$
\left[[x, y]^{\prime}, z\right]^{\prime}+\left[[y, z]^{\prime}, x\right]^{\prime}+\left[[z, x]^{\prime}, y\right]^{\prime}=0
$$

Clearly, $[\beta(x), \alpha(y)]=-[\beta(y), \alpha(x)]$, So we have

$$
[x, y]^{\prime}=-[y, x]^{\prime}
$$

It follows immediately that $\left(A,[\cdot, \cdot]^{\prime \prime}\right)$ is also a Lie algebra when $\alpha, \beta$ are involution.

Let $(A, \mu)$ be a commutative associative algebra, $\alpha, \beta: A \rightarrow A$ be two commuting algebra morphism, and $D: A \rightarrow A$ be an even derivation with $D \alpha=\alpha D, D \beta=\beta A$. Consider the following operation on $A$ :

$$
x \star y=\alpha(x) D(\beta(y)), \quad \text { for any } x, y \in A \text {. }
$$


Next we check equation (2.2), on one hand,

$$
\begin{aligned}
(x \star y) \star \alpha(z) & =(\alpha(x) \Delta(\beta(y))) \star \alpha(z) \\
& =\left(\alpha^{2}(x) D(\alpha \beta(y))\right) D(\alpha \beta(z)) \\
& =\alpha^{2}(x)(D(\alpha \beta(y)) D(\alpha \beta(z))),
\end{aligned}
$$

on the other hand,

$$
\begin{aligned}
(x \star z) \star \alpha(y) & =(\alpha(x) \Delta(\beta(z))) \star \alpha(y) \\
& =\left(\alpha^{2}(x) D(\alpha \beta(z))\right) D(\alpha \beta(y)) \\
& =\alpha^{2}(x)(D(\alpha \beta(z)) D(\alpha \beta(y))) \\
& =\alpha^{2}(x)(D(\alpha \beta(y)) D(\alpha \beta(z))),
\end{aligned}
$$

hence we have

$$
(x \star y) \star \alpha(z)=(x \star z) \star \alpha(y)
$$

Similarly, we have

$$
\begin{aligned}
& (\beta(x) \star \alpha(y)) \star \beta(z)-\alpha \beta(x)(\alpha(y) z) \\
= & \alpha^{2} \beta(x)\left(D\left(\alpha^{2} \beta(y)\right) D\left(\beta^{2}(z)\right)\right)-\alpha^{2} \beta(x)\left(D\left(\alpha^{2} \beta(y) D(\beta(z))\right),\right.
\end{aligned}
$$

and

$$
\begin{aligned}
& (\beta(y) \star \alpha(x)) \star \beta(z)-\alpha \beta(y)(\alpha(x) z) \\
= & \alpha^{2} \beta(y)\left(D \alpha^{2} \beta(x) D\left(\beta^{2}(z)\right)\right)-\alpha^{2} \beta(y)\left(D\left(\alpha^{2} \beta(x) D(\beta(z))\right) .\right.
\end{aligned}
$$

Now from the discussions above, we obtain

Theorem 2.8. Let $(A, \mu)$ be a commutative associative algebra, $\alpha, \beta: A \rightarrow A$ be two commuting algebra morphism, and $D: A \rightarrow A$ be an even derivation with $D \alpha=\alpha D, D \beta=$ $\beta A$. Then $(A, \mu, \alpha, \beta)$ is a BiHom-Novikov algebra, where $\star$ is defined by $(2.8)$.

Definition 2.9. Let $(A, \star, \alpha, \beta)$ be a BiHom-algebra and let $\lambda \in k$. If a linear map $P: A \rightarrow A$ stisfies

$$
P(x) \star P(y)=P(P(x) \star y+x \star P(y)+\lambda x \star y), \quad \text { for any } x, y \in A .
$$

Then $P$ is called a Rota-Baxter operator of weight $\lambda$ and $(A, \star, \alpha, \beta, P)$ is called a RotaBaxter Hom-algebra of weight $\lambda$.

Theorem 2.10. Let $(A, \star, \alpha, \beta, P)$ be a Rota-Baxter BiHom-Novikov algebra of weight $\lambda$ and $P$ an even linear map. Assume that $\alpha, \beta$ and $P$ commute, define a new multiplication on $A$ by

$$
x \circ y=P(x) \star y+x \star P(y)+\lambda x \star y, \quad \text { for any } x, y \in A \text {. }
$$


Then $(A, \circ, \alpha, \beta, P)$ is a BiHom-Novikov algebra.

Proof. The multiplication of $\alpha, \beta$ with respect to $\circ$ follows from the multiplication of $\alpha, \beta$ with respect to $\star$ and the hypothesis $P \alpha=\alpha P, P \beta=\beta P$. We only check equation (2.2) and (2.3 is left to the reader.) For any $x, y, z \in A$, on one hand, we have,

$$
\begin{aligned}
& (x \circ y) \circ \alpha(z) \\
= & P(P(x) \star y+x \star P(y)+\lambda x \star y) \star \alpha(z)+(P(x) \star y+x \star P(y)+\lambda x \star y) \star P(\alpha(z)) \\
& +\lambda(P(x) \star y+x \star P(y)+\lambda x \star y) \star \alpha(z) \\
= & (P(x) \star P(y)) \star \alpha(z)+(P(x) \star y) \star \alpha(P(z))+(x \star P(y)) \star \alpha(P(z))+\lambda(x \star y) \star \alpha(P(z)) \\
& \lambda(P(x) \star y) \star \alpha(z)+\lambda(x \star P(y)) \star \alpha(z)+\lambda^{2}(x \star y) \star \alpha(z) \\
= & (P(x) \star z) \star \alpha(P(y))+(P(x) \star P(z)) \star \alpha(P(y))+(x \star P(z)) \star \alpha(P(y))+\lambda(x \star P(z)) \star \alpha(y) \\
& \lambda(P(x) \star z) \star \alpha(y)+\lambda(x \star z) \star \alpha(P(y))+\lambda^{2}(x \star z) \star \alpha(y) .
\end{aligned}
$$

On the other hand, we have

$$
\begin{aligned}
& (x \circ z) \circ \alpha(y) \\
= & (P(x) \star z) \star \alpha(P(y))+(P(x) \star P(z)) \star \alpha(P(y))+(x \star P(z)) \star \alpha(P(y))+\lambda(x \star P(z)) \star \alpha(y) \\
& \lambda(P(x) \star z) \star \alpha(y)+\lambda(x \star z) \star \alpha(P(y))+\lambda^{2}(x \star z) \star \alpha(y) .
\end{aligned}
$$

Hence, the conclusion holds.

\section{Quadratic BiHom-Novikov algebras}

In this section, we extend the notions of quadratic Novikov algebras and quadratic Hom-Novikov algebras to quadratic BiHom-Novikov algebras and provide some properties. we always assume that the structure maps $\alpha$ and $\beta$ are bijective.

Definition 3.1. Let $(A,[\cdot, \cdot], \alpha, \beta)$ be a BiHom-Lie algebra and $B: A \times A \rightarrow k$ be a bilinear form on $A$.

(1) $B$ is said nondegenerate if

$$
A^{\perp}=\{x \in A \mid B(x, y)=0, \forall y \in A\}=0 .
$$

(2) $B$ is said symmetric if

$$
B(x, y)=B(y, x), \text { for any } x, y \in A .
$$

(3) $B$ is said $\alpha \beta$-invariant if

$$
B([\beta(x), \alpha(y)], \alpha(z))=B(\alpha(x),[\beta(y), \alpha(z)]), \text { for any } x, y, z \in A \text {. }
$$


Definition 3.2. A quadratic BiHom-Lie algebra is a quintuple $(A,[\cdot, \cdot], \alpha, \beta, B)$ such that $(A,[\cdot, \cdot], \alpha, \beta)$ is a BiHom-Lie algebra with a symmetric invariant nondegenerate bilinear form $B$ satisfying

$$
B(\alpha(x), y)=B(x, \alpha(y)), B(\beta(x), y)=B(x, \beta(y)) \quad \text { for any } x, y \in A .
$$

We can define quadratic BiHom-Novikov algebras as follows.

Definition 3.2. A quadratic BiHom-Novikov algebra is a quintuple $(A, \mu, \alpha, \beta, B)$ is a BiHom-Novikov algebra $(A, \mu, \alpha, \beta)$ with a symmetric invariant nondegenerate bilinear form $B$ satisfying

$$
B(\alpha(x), \beta(y) \alpha(z))=B(\beta(x) \alpha(y), \alpha(z)), \quad \text { for any } x, y, z \in A .
$$

Proposition 3.3. Let $(A, \mu, \alpha, \beta, B)$ be a quadratic BiHom-Novikov algebra and $(A,[\cdot, \cdot], \alpha, \beta)$ be the sub-adjacent BiHom-Lie algebra of $A$, if

$$
B(\alpha(x), y)=B(x, \alpha(y)), B(\beta(x), y)=B(x, \beta(y)), \quad \text { for any } x, y \in A .
$$

Then $\left(A,[\cdot, \cdot], \alpha, \beta, B_{\alpha, \beta}\right)$ is a quadratic BiHom-Lie algebra, where $B_{\alpha, \beta}(x, y)=B(\alpha(x), y)$.

Proof. Since $B$ is a nondegenerate bilinear form, $B_{\alpha}$ is a nondegenerate bilinear form on $A$. For all $x, y, z \in A$, using the properties of $B$, we have

$$
\begin{aligned}
B_{\alpha}([\beta(x), \alpha(y)], \alpha(z)) & =B(\alpha[\beta(x), \alpha(y)], \alpha(z)) \\
& =B\left([\beta(x), \alpha(y)], \alpha^{2}(z)\right) \\
& =B\left(\beta(x) \alpha(y), \alpha^{2}(z)\right)-B\left(\beta(y) \alpha(x), \alpha^{2}(z)\right) \\
& =B\left(\alpha^{2}(x), \beta(y) \alpha(z)\right)-B\left(\alpha^{2}(x), \beta(z) \alpha(y)\right) \\
& =B\left(\alpha^{2}(x),[\beta(y), \alpha(z)]\right) \\
& =B_{\alpha}(\alpha(x),[\beta(y), \alpha(z)]) .
\end{aligned}
$$

Hence, $B_{\alpha}$ is invariant. Using symmetry of $B$ and equation (3.3), we have

$$
B_{\alpha}(x, y)=B(\alpha(x), y)=B(y, \alpha(x))=B(\alpha(y), x)=B_{\alpha}(y, x) .
$$

which proves $B_{\alpha}$ is symmetric. Using equation (3.3) again, we have

$$
\begin{gathered}
B_{\alpha}(\alpha(x), y)=B(\alpha(\alpha(x)), y)=B(\alpha(x), \alpha(y))=B_{\alpha}(x, \alpha(y)), \\
B_{\alpha}(\beta(x), y)=B(\alpha(\beta(x)), y)=B(\alpha(x), \beta(y))=B_{\alpha}(x, \beta(y)) .
\end{gathered}
$$

Corollary 3.4. Let $(A, \mu, \alpha, \beta, B)$ be a quadratic BiHom-Novikov algebra and $\alpha, \beta$ satisfying equation $(3.4)$ and $(A,[\cdot, \cdot])$ be the sub-adjacent Lie algebra, define a new multiplication on $A$ by $[x, y]^{\prime}=[\alpha(x), \beta(y)]$. Then $\left(A,[\cdot, \cdot]^{\prime}, \alpha, \beta, B_{\alpha}\right)$ is a quadratic Hom-Lie algebra, where $B_{\alpha, \beta}(x, y)=B(\alpha(x), y)$. 
Proof. Using the similar arguments as those in the proof of Proposition 3.3, we get $B_{\alpha}$ is a symmetric nondegenerate bilinear form with equation (3.1) satisfied. It remains to show that $B_{\alpha}$ is invariant. For all $x, y, z \in A$, using invariance and symmetry of $B$, we have

$$
\begin{aligned}
B_{\alpha}\left([\beta(x), \alpha(y)]^{\prime}, \alpha(z)\right) & =B(\alpha[\alpha \beta(x), \alpha \beta(y)], \alpha(z)) \\
& =B\left([\alpha \beta(x), \alpha \beta(y)], \alpha^{2}(z)\right) \\
& =B\left(\alpha \beta(x) \alpha \beta(y), \alpha^{2}(z)\right)-B\left(\beta^{2}(y) \alpha^{2}(x), \alpha^{2}(z)\right) \\
& =B\left(\alpha^{2}(x), \alpha \beta(y) \alpha \beta(z)\right)-B\left(\alpha^{2}(x), \beta(z) \alpha(y)\right) \\
& =B\left(\alpha^{2}(x),[\alpha \beta(y), \alpha \beta(z)]\right) \\
& =B_{\alpha}\left(\alpha(x),[\beta(y), \alpha(z)]^{\prime}\right) .
\end{aligned}
$$

Proposition 3.5. Let $(A, \mu, \alpha, \beta, B)$ be a quadratic BiHom-Novikov algebra, where $\alpha, \beta$ are involution satisfying equation (3.3), define a new multiplication on $A$ by $x \star y=$ $\alpha(x) \star \beta(y)$ Then $(A, \star, \alpha, \beta, B)$ is a quadratic Novikov algebra.

Proof. $(A, \mu, \alpha, \beta, B)$ is a BiHom-Novikov algebra by Proposition 2.5. It suffices to show that $B$ is invariant under the operation $\star$. For all $x, y, z \in A$, we have

$$
\begin{aligned}
B(\alpha(x), \beta(y) \star \alpha(z)) & =B(\alpha(x), \alpha \beta(y) \alpha \beta(z)) \\
=B(\alpha \beta(x) \alpha \beta(y), \alpha(z)) & =B(\beta(x) \star \alpha(y), \alpha(z)),
\end{aligned}
$$

which completes the proof.

Let $(A, \mu, \alpha, \beta)$ be a BiHom-Novikov algebra, whose center is denoted by $Z(A)$ and defined by

$$
Z(A)=\{x \in A \mid x y=y x=0, \forall y \in A\} .
$$

Let $\left(A,[\cdot, \cdot], \alpha^{\prime}, \beta^{\prime}\right)$ be a BiHom-Lie algebra. The lower central series of $A$ is defined as usual, i.e., $A^{0}=A, A^{i}=\left[A, A^{i-1}\right], \forall i \geq 1$. We call $A$ is $i$-step nilpotent if $A^{i}=0$ and $A^{i-1} \neq 0$. The center of the Hom-Lie superalgebra is denoted by $C(A)$ and defined by

$$
C(A)=\{x \in A \mid[x, y]=0, \forall y \in A\}
$$

Theorem 3.6. Let $(A, \mu, \alpha, \beta, B)$ be a quadratic BiHom-Novikov algebra, and HLie $(A)$ be the sub-adjacent BiHom-Lie algebra. Then $[\beta(x), \alpha(y)] \in Z(A)$, for any $x, y \in H \operatorname{Lie}(A)$. As a consequence, $H \operatorname{Lie}(A)$ is 2-step nilpotent.

Proof. For any $x, y, z \in A$, we have

$$
\begin{aligned}
\alpha(x)\left[y^{\prime}, z^{\prime}\right]=\alpha(x)[\beta(y), \alpha(z)] & =\alpha(x)(\beta(y) \alpha(z))-\alpha(x)(\beta(y) \alpha(z)) \\
& =(x \beta(y)) \alpha \beta(z)-(x \beta(y) \alpha \beta(z) \\
& =(x \beta(y)) \alpha \beta(z)-(x \beta(y) \alpha \beta(z) \\
& =0 .
\end{aligned}
$$


Using Equation (3.2), we have

$$
B\left(\left[y^{\prime}, z^{\prime}\right] \alpha(d), \alpha^{2}(x)\right)=B\left(\alpha(x) \alpha \beta^{-1}\left[y^{\prime}, z^{\prime}\right], \alpha^{2}(d)\right)=0
$$

which implies $[x, y] \in Z(A)$ since $\alpha, \beta$ are automorphism and $B$ is nondegenerate. Hence, we have $[H \operatorname{Lie}(A), H \operatorname{Lie}(A)] \in Z(A)$. Obviously, $Z(A) \in C(H \operatorname{Lie}(A))$. Then it follows that $H \operatorname{Lie}(A)$ is 2-step nilpotent.

\section{1-parameter formal deformations of BiHom-Novikov al- gebras}

Definition 4.1. Let $(A, *, \alpha, \beta)$ be a regular BiHom-Novikov algebra. If an $n$-linear map $f: A \times \cdots \times A \rightarrow A$ satisfies

$$
\begin{aligned}
\alpha\left(f\left(x_{1}, \cdots, x_{n}\right)\right) & =f\left(\alpha\left(x_{1}\right), \cdots, \alpha\left(x_{n}\right)\right), \\
\beta\left(f\left(x_{1}, \cdots, x_{n}\right)\right) & =f\left(\beta\left(x_{1}\right), \cdots, \beta\left(x_{n}\right)\right),
\end{aligned}
$$

then $f$ is called an $n$-BiHom-cochain on $A$. Denote by $C_{\alpha, \beta}^{n}(A, A)$ the set of all $n$-Homcochains, $\forall n \geq 1$.

Definition 4.2. For $n=1,2$, the coboundary operator $\delta_{h o m}^{n}: C_{\alpha, \beta}^{n}(A, A) \rightarrow C_{\alpha, \beta}^{n+1}(A, A)$ is defined as follows

$$
\begin{aligned}
& \delta_{\text {hom }}^{1} f\left(x_{1}, x_{2}\right)=x_{1} * f\left(x_{2}\right)+f\left(x_{1}\right) * x_{2}-f\left(\alpha^{-1} \beta\left(x_{1}\right) * x_{2}\right), \\
& \delta_{\text {hom }}^{2} f\left(x_{1}, x_{2}, x_{3}\right)=f\left(\beta\left(x_{1}\right), \alpha^{-1} \beta\left(x_{2}\right) * x_{3}\right)-f\left(\alpha^{-1} \beta\left(x_{1}\right) * x_{2}, \beta\left(x_{3}\right)\right) \\
& -f\left(\beta\left(x_{2}\right), \alpha^{-1} \beta\left(x_{1}\right) * x_{3}\right)+f\left(\alpha^{-1} \beta\left(x_{2}\right) * x_{1}, \beta\left(x_{3}\right)\right) \\
& +\beta\left(x_{1}\right) * f\left(x_{2}, x_{3}-f\left(x_{1}, x_{2}\right) * \beta\left(x_{3}\right)\right. \\
& +\beta\left(x_{2}\right) * f\left(x_{1}, x_{3}\right)+f\left(x_{2}, x_{1}\right) * \beta\left(x_{3}\right) .
\end{aligned}
$$

It is not difficult to verify that $\delta_{h o m}^{1}, \delta_{h o m}^{2}$ satisfies (4.1). Thus, the coboundary operator $\delta_{h o m}^{n}$ is well-defined.

Theorem 4.3. The coboundary operator $\delta_{h o m}^{1}, \delta_{h o m}^{2}$ defined above satisfies $\delta_{h o m}^{2} \delta_{h o m}^{1}=0$.

Proof. Similar to [28] and left to the reader.

For $n=1,2$, the map $f \in C_{\alpha, \beta}^{n}(A, A)$ is called an $n$-Hom-cocycle if $\delta_{\text {hom }}^{n} f=0$. We denote $Z_{\alpha, \beta}^{n}(A, A)$ the subspace spanned by $n$-BiHom-cocycles and $B_{\alpha, \beta}^{n}(A, A)=$ $\delta_{\text {hom }}^{n-1} C_{\alpha, \beta}^{n-1}(A, A)$. Since $\delta_{h o m}^{2} \delta_{h o m}^{1}=0, B_{\alpha, \beta}^{n}(A, A)$ is a subspace of $Z_{\alpha, \beta}^{n}(A, A)$. Hence we can define a cohomology space $H_{\alpha, \beta}^{2}(A, A)$ of $(A, *, \alpha, \beta)$ as the factor space $Z_{\alpha, \beta}^{2}(A, A) / B_{\alpha, \beta}^{2}(A, A)$.

Let $(A, *, \alpha, \beta)$ be a regular BiHom-Novikov algebra and $K[[t]]$ be the ring of formal power series over $\mathbb{K}$. Suppose that $A[[t]]$ is the set of formal power series over $A$. Then 
for an $\mathbb{K}$-bilinear map $f: A \times A \rightarrow A$, it is natural to extend it to be a $\mathbb{K}[[t]]$-bilinear map $f: A[[t]] \times A[[t]] \rightarrow A[[t]]$ by

$$
f\left(\sum_{i \geq 0} x_{i} t^{i}, \sum_{j \geq 0} y_{j} t^{j}\right)=\sum_{i, j \geq 0} f\left(x_{i}, y_{j}\right) t^{i+j} .
$$

Definition 4.4. Let $(A, *, \alpha, \beta)$ be a regular BiHom-Novikov algebra over $\mathbb{K}$. A 1parameter formal deformation of $(A, *, \alpha, \beta)$ is a formal power series $g_{t}: A[[t]] \times A[[t]] \rightarrow$ $A[[t]]$ of the form

$$
g_{t}(x, y)=\sum_{i \geq 0} G_{i}(x, y) t^{i}=G_{0}(x, y)+G_{1}(x, y) t+G_{2}(x, y) t^{2}+\cdots
$$

where each $G_{i}$ is an even $\mathbb{K}$-bilinear map $G_{i}: A \times A \rightarrow A$ and $G_{0}(x, y)=x * y$ such that the following identities hold

$$
\begin{aligned}
& g_{t}(\alpha(x), \alpha(y))=\alpha \circ g_{t}(x, y), \\
& g_{t}(\beta(x), \beta(y))=\beta \circ g_{t}(x, y), \\
& g_{t}\left(g_{t}(x, y), \alpha(z)\right)=g_{t}\left(g_{t}(x, z), \alpha(y)\right), \\
& g_{t}\left(\alpha \beta(x), g_{t}(\alpha(y), z)\right)-g_{t}\left(g_{t}(\beta(x) \alpha(y)), \beta(z)\right) \\
& -g_{t}\left(\alpha \beta(y), g_{t}(\alpha(x), z)\right)+g_{t}\left(g_{t}(\beta(y), \alpha(x)), \beta(z)\right)=0 .
\end{aligned}
$$

Conditions (4.2)-(4.5) are called the deformation equations of a regular BiHom-Novikov algebra.

Note that $A[[t]]$ is a module over $K[[t]]$ and $g_{t}$ defines the bilinear multiplication on $A[[t]]$ such that $A_{t}=\left(A[[t]], g_{t}, \alpha, \beta\right)$ is a BiHom-Novikov algebra. Now we investigate the deformation equations (4.2)-(4.5).

Conditions (4.2)-(4.5) are equivalent to the following equations

$$
\begin{aligned}
& G_{i}(\alpha(x), \alpha(y))=\alpha \circ G_{i}(x, y), \\
& G_{i}(\beta(x), \beta(y))=\beta \circ G_{i}(x, y), \\
& G_{i}\left(G_{j}(x, y), \alpha(z)\right)=G_{i}\left(G_{j}(x, z), \alpha(y)\right), \\
& G_{i}\left(\alpha \beta(x), G_{j}(\alpha(y), z)\right)-G_{i}\left(G_{j}(\beta(x) \alpha(y)), \beta(z)\right) \\
& -G_{i}\left(\alpha \beta(y), G_{j}(\alpha(x), z)\right)+G_{i}\left(G_{j}(\beta(y), \alpha(x)), \beta(z)\right)=0 .
\end{aligned}
$$

For $n=0$, this means $A=A_{0}$ is a BiHom-Novikov algebra. For $n=1$, we obtain some 
results for $G_{1}$ :

$$
\begin{aligned}
& G_{i}(\alpha(x), \alpha(y))=\alpha \circ G_{i}(x, y), \\
& G_{i}(\beta(x), \beta(y))=\beta \circ G_{i}(x, y), \\
& G_{1}(x * y, \alpha(z))+G_{1}(x, y) * \alpha(z)=G_{1}(x * z, \alpha(y))+G_{1}(x, z) * \alpha(y), \\
& \alpha \beta(x) * G_{1}(\alpha(y), z)-G_{1}(\alpha(x), \alpha(y)) * \beta(z)-\alpha \beta(y) * G_{1}(\alpha(x), z)+G_{1}(\alpha(y) * \alpha(x)) * \beta(z) \\
& +\alpha \beta(x) * G_{1}(\alpha(y), z)-G_{1}(\beta(x), \alpha(y)) * \beta(z)-\alpha \beta(y) * G_{1}(\alpha(x), z)+G_{1}(\beta(y), \alpha(x)) * \beta(z)=0 .
\end{aligned}
$$

Example 4.5. By equation (2.8) with a fixed derivation $D$, we obtain a BiHom-Novikov algebra $(A, \star, \alpha, \beta)$. Now we define a new multiplication $*_{\xi}$ on $A$ as

$$
x *_{\xi} y=\alpha(x) D(\beta(y))+\xi x y .
$$

for all $x, y \in A, \xi \in \mathbb{F}$. Then $(A, \star, \alpha, \beta)$ is an infinitesimal deformation BiHom-Novikov algebra, we let $G_{1}(x, y)=\alpha(x) \beta(y)$, and it is straightforward to check that equations (4.10)-(4.13) hold.

Hence, we have the following result.

Theorem 4.6. Let $(A, \mu)$ be a commutative algebra, $\alpha, \beta: A \rightarrow A$ be two commuting algebra morphism, and $D: A \rightarrow A$ be an even derivation such that $D \alpha=\alpha D, D \beta=\beta D$. Then $\left(A, *_{\xi}, \alpha, \beta\right)$ is a BiHom-Novikov algebra, where $*_{\xi}$ is defined as

$$
x *_{\xi} y=\alpha(x) D(\beta(y))+\xi x y,
$$

for all $x, y \in A, \xi \in \mathbb{K}$.

For two $\mathbb{K}$-bilinear maps $f, g: A \times A \rightarrow A$, define a map: $f \circ_{\alpha, \beta} g: A[[t]] \times A[[t]] \times A[[t]] \rightarrow$ $A[[t]]$ by

$$
\begin{aligned}
f \circ_{\alpha, \beta} g(x, y, z)= & f(\alpha \beta(x), g(\alpha(y), z))-f(g(\beta(x) \alpha(z)), \beta(y)) \\
& -f(\alpha \beta(y), g(\alpha(x), z))+f(g(\beta(y), \alpha(x)), \beta(z))
\end{aligned}
$$

Using equations (4.8) and (4.9), we have

$$
\sum_{i+j=n} G_{i} \circ_{\alpha, \beta} G_{j}=0
$$

For $n=1$, we have

$$
G_{0} \circ_{\alpha, \beta} G_{1}+G_{1} \circ_{\alpha, \beta} G_{0}=0 .
$$

For any $n \geq 2$, we have

$-\left(G_{0} \circ_{\alpha, \beta} G_{n}+G_{n} \circ_{\alpha, \beta} G_{0}\right)=G_{1} \circ_{\alpha, \beta} G_{n-1}+G_{2} \circ_{\alpha, \beta} G_{n-2}+\cdots+G_{n-1} \circ_{\alpha, \beta} G_{0}$. 
By (4.5)and (4.6) it follows that $G_{i} \in C_{\alpha, \beta}^{2}(A, A)$. It can also be verified that $G_{i}{ }^{\circ}{ }_{\alpha, \beta}$ $G_{j} \in C_{\alpha, \beta}^{3}(A, A)$. In general, if $f, g \in C_{\alpha, \beta}^{2}(A, A)$, then $f \circ_{\alpha, \beta} g \in C_{\alpha, \beta}^{3}(A, A)$. Note that the definition of coboundary operator, which implies $\delta_{h o m}^{2} G_{n}=G_{0} \circ_{\alpha, \beta} G_{n}+G_{n} \circ_{\alpha, \beta} G_{0}$ for $n=0,1,2 \cdots$. Hence (4.16) and (4.17) can be rewritten as

$$
\begin{array}{r}
\delta_{\text {hom }}^{2} G_{1}=0, \\
-\delta_{h o m}^{2} G_{n}=G_{1} \circ_{\alpha, \beta} G_{n-1}+G_{2} \circ_{\alpha, \beta} G_{n-2}+\cdots+G_{n-1} \circ_{\alpha, \beta} G_{0} .
\end{array}
$$

Then $G_{1}$ is a 2 -BiHom-cocycle.

Definition 4.7. Let $(A, *, \alpha, \beta)$ be a regular BiHom-Novikov algebra. Suppose that $g_{t}(x, y)=G_{0}\left(\alpha^{-1} \beta(x), y\right)+\sum_{i>0} G_{i}(x, y) t^{i}$ and $g_{t}^{\prime}(x, y)=\sum_{i \geq 0} G_{i}^{\prime}(x, y) t^{i}$ are two 1parameter formal deformations of $(A, *, \alpha, \beta)$. They are called equivalent, denoted by $g_{t} \sim g_{t}^{\prime}$, if there is a formal isomorphism of $\mathbb{K}[[t]]$-modules

$$
\phi_{t}(x)=\sum_{i \geq 0} \phi_{i}(x) t^{i}:\left(A[[t]], g_{t}, \alpha, \beta\right) \rightarrow\left(A[[t]], g_{t}^{\prime}, \alpha, \beta\right)
$$

where each $\phi_{i}: T \rightarrow T$ is a $\mathbb{K}$-linear map and $\phi_{0}=i d_{A}$, satisfying

$$
\begin{array}{r}
\phi_{t} \circ \alpha=\alpha \circ \phi_{t}, \\
\phi_{t} \circ \beta=\beta \circ \phi_{t}, \\
\phi_{t} \circ g_{t}(x, y)=g_{t}^{\prime}\left(\phi_{t}(x), \phi_{t}(y)\right) .
\end{array}
$$

When $G_{1}=G_{2}=\cdots=0, g_{t}=G_{0}$ is said to be the null deformation. A 1-parameter formal deformation $g_{t}$ is called trivial if $g_{t} \sim G_{0}$. A BiHom-Novikov algebra $(T,[\cdot, \cdot, \cdot], \alpha, \beta)$ is called analytically rigid, if every 1-parameter formal deformation $g_{t}$ is trivial.

Theorem 4.8. Let $g_{t}(x, y)=G_{0}\left(\alpha^{-1} \beta(x), y\right)+\sum_{i>0} G_{i}(x, y) t^{i}$ and $g_{t}^{\prime}(x, y)=\sum_{i \geq 0} G_{i}^{\prime}(x, y) t^{i}$ be equivalent 1-parameter formal deformations of $(A, *, \alpha, \beta)$. Then $G_{1}$ and $G_{1}^{\prime}$ belong to the same cohomology class in $H_{\alpha, \beta}^{2}(A, A)$.

Proof. Suppose that $\phi_{t}(x)=\sum_{i \geq 0} \phi_{i}(x) t^{i}$ is the formal $\mathbb{K}[[t]]$-module isomorphism such that $\phi_{t} \circ \alpha=\alpha \circ \phi_{t}, \phi_{t} \circ \beta=\beta \circ \phi_{t}$ and

$$
\sum_{i \geq 0} \phi_{i}\left(G_{0}\left(\alpha^{-1} \beta(x), y\right)+\sum_{j>0} G_{j}(x, y) t^{j}\right) t^{i}=\sum_{i \geq 0} G_{i}^{\prime}\left(\sum_{k \geq 0} \phi_{k}(x) t^{k}, \sum_{k \geq 0} \phi_{l}(y) t^{l}\right) t^{i} .
$$

It follows that

$$
\sum_{i+j=n} \phi_{i}\left(G_{0}\left(\alpha^{-1} \beta(x), y\right)+\sum_{j>0} G_{j}(x, y)\right) t^{i+j}=\sum_{i+k+l=n} G_{i}^{\prime}\left(\phi_{k}(x), \phi_{l}(y)\right) t^{i+k+l} .
$$

In particular,

$$
\left.\sum_{i+j=1} \phi_{i}\left(G_{0}\left(\alpha^{-1} \beta(x), y\right)+G_{j}(x, y)\right)\right) t^{i+j}=\sum_{i+k+l=1} G_{i}^{\prime}\left(\phi_{k}(x), \phi_{l}(y)\right) t^{i+k+l} .
$$


that is,

$$
G_{1}(x, y)+\phi_{1}\left(\alpha^{-1} \beta(x) * y\right)=\phi_{1}(x) * y+x * \phi_{1}(y)+G_{1}^{\prime}(x, y) .
$$

Then $G_{1}-G_{1}^{\prime} \in B_{\alpha, \beta}^{2}(A, A)$.

Theorem 4.9. Suppose that $(A, *, \alpha, \beta)$ is a regular BiHom-Novikov algebra such that $H_{\alpha, \beta}^{2}(A, A)=0$. Then $(A, *, \alpha, \beta)$ is analytically rigid.

Proof. Let $g_{t}$ be a 1-parameter formal deformation of $(A, *, \alpha, \beta)$. Suppose that $g_{t}=G_{0}+\sum_{i \geq n} G_{i} t^{i}$. Then

$$
\delta_{\text {hom }}^{2} G_{n}=G_{1} \circ_{\alpha, \beta} G_{n-1}+G_{2} \circ_{\alpha, \beta} G_{n-2}+\cdots+G_{n-1} \circ_{\alpha, \beta} G_{0}=0,
$$

that is $G_{n} \in Z_{\alpha, \beta}^{2}(A, A)=B_{\alpha, \beta}^{2}(A, A)$. It follows that there exists $f_{n} \in C_{\alpha, \beta}^{1}(A, A)$ such that $G_{n}=\delta_{\text {hom }}^{1} f_{n}$.

Let $\phi_{t}=i d_{A}-f_{n} t^{n}:\left(A[[t]], g_{t}^{\prime}, \alpha, \beta\right) \rightarrow\left(A[[t]], g_{t}, \alpha, \beta\right)$. Note that

$$
\phi_{t} \circ \sum_{i \geq 0} f_{n}^{i} t^{i n}=\sum_{i \geq 0} f_{n}^{i} t^{i n} \circ \phi_{t}=i d_{A[[t]]} .
$$

Then $\phi_{t}$ is a linear isomorphism. Moreover, $\phi_{t} \circ \alpha=\alpha \circ \phi_{t}$ and $\phi_{t} \circ \beta=\beta \circ \phi_{t}$.

Now we consider $g_{t}^{\prime}(x, y)=\phi_{t}^{-1} g_{t}\left(\phi_{t}(x), \phi_{t}(y)\right)$. It is straightforward to prove that $g_{t}^{\prime}$ is a 1-parameter formal deformation of $(A, *, \alpha, \beta)$ and $g_{t} \sim g_{t}^{\prime}$, Suppose that $g_{t}^{\prime}(x, y)=$ $G_{0}^{\prime}\left(\alpha^{-1} \beta(x), y\right)+\sum_{i>0} G_{i}^{\prime}(x, y) t^{i}$. Then

$$
\begin{aligned}
& \left(i d_{T}-f_{n} t^{n}\right)\left(G_{0}^{\prime}\left(\alpha^{-1} \beta(x), y\right)+\sum_{i>0} G_{i}^{\prime}(x, y) t^{i}\right) \\
= & \left(G_{0}^{\prime}\left(\alpha^{-1} \beta(x), y\right)+\sum_{i \geq n} G_{i}^{\prime}(x, y) t^{i}\right)\left(x-f_{n}(x) t^{n}\right)\left(y-f_{n}(y) t^{n}\right),
\end{aligned}
$$

i.e.,

$$
\begin{aligned}
& G_{0}^{\prime}\left(\alpha^{-1} \beta(x), y\right)+\sum_{i>0} G_{i}^{\prime}(x, y) t^{i}-f_{n}\left(G_{0}^{\prime}\left(\alpha^{-1} \beta(x), y\right)+\sum_{i>0} G_{i}^{\prime}(x, y) t^{i}\right) \\
= & \alpha^{-1} \beta(x) * y-\left(f_{n}(x) * y+x * f_{n}(y)\right) t^{n}+f_{n}(x) * f_{n}(y) t^{2 n} \\
& +\sum_{i \geq n} G_{i}(x, y) t^{i}-\sum_{i \geq n}\left(G_{i}\left(f_{n}(x), y\right)+G_{i}\left(x, f_{n}(y)\right) t^{i+n}+\sum_{i \geq n} G_{i}\left(f_{n}(x), f_{n}(y)\right) t^{i+2 n} .\right.
\end{aligned}
$$

Then we have $G_{1}^{\prime}=G_{2}^{\prime}=\ldots=G_{n-1}^{\prime}=0$ and

$$
G_{n}^{\prime}(x, y)-f_{n}\left(\alpha^{-1} \beta(x) * y\right)=-\left(f_{n}(x) * y+x * f_{n}(y)\right)+G_{n}(x, y) .
$$

Hence $G_{n}^{\prime}=G_{n}-\delta^{1} f_{n}=0$ and $g_{t}^{\prime}(x, y)=G_{0}^{\prime}\left(\alpha^{-1} \beta(x), y\right)+\sum_{i>0} G_{i}^{\prime}(x, y) t^{i}$. By induction, this procedure ends with $g_{t} \sim G_{0}$, i.e., $(A, *, \alpha, \beta)$. is analytically rigid.

\section{ACKNOWLEDGEMENT}

The paper is partially supported by the NSF of China (No. 11761017), the Fund of Science and Technology Department of Guizhou Province (No. [2016]1021), the Key University Science Research Project of Anhui Province (Nos. KJ2015A294, KJ2014A183 and KJ2016A545), the NSF of Chuzhou University (Nos. 2014PY08 and 2015qd01). 


\section{REFERENCES}

[1] K. Abdaoui, A. Ben Hassine and A. Makhlouf, BiHom-Lie colour algebras structures, arxiv: $1706.02188 \mathrm{v} 1(2017)$

[2] N. Aizawa, and H. Sato, $q$-deformation of the Virasoro algebra with central extension, Phys. Lett. B, 256(1991), 185-190.

[3] L. Auslander, Simply transitive groups of affine motions, Amer. J. Math. 99 (1977), $809 \mathrm{C} 826$.

[4] A. A. Balinskii, S. P. Novikov, Poisson brackets of hydrodynamic type, Frobenius algebras and Lie algebras, Sov. Math. Dokl. 32(1985), 228-231.

[5] C. Bai, D. Meng. The realization of non-transitive Novikov algebras. J. Phys. A 34 (2001), 6435-6442.

[6] C. Bai, D. Meng, A Lie algebraic approach to Novikov algebras, J. Geom. Phys. 45 (2003), 218-230.

[7] M. Bordemann, Nondegenerate invariant bilinear forms on nonassociate algebras, Acta. Math. Univ. Comenianae 66 (1997), 151-201.

[8] M. Chaichian, D. Ellinas and Z. Popowicz, Quantum conformal algebra with central extension, Phys. Lett. B, 248(1990), 95-99.

[9] Y. Cheng and H. Qi, Representations of BiHom-Lie algebras, arXiv:1610.04302 v1(2016).

[10] B. A. Dubrovin, S. P. Novikov, Hamiltonian formalism of one-dimensional systems of hydrodynamic type and the Bogolyubov-Whitham averaging method,Sov. Math. Dokl. 27 (1983), 665-669.

[11] V. T. Filipov, A class of simple nonassociative algebras, Mat. Zametki 45 (1989), 101-105.

[12] I. M. Gel'fand, I. Ya. Dorfman, Hamiltonian operators and algebraic structures related to them, Funct. Anal. Appl. 13(1979), 248-262

[13] M. Gerstenhaber, On the deformation of rings and algebras. Ann. of Math. (2) 79 (1964), 59-103.

[14] M. Gerstenhaber, On the deformation of rings and algebras. II. Ann. of Math. 84 (1966), 1-19. 
[15] M. Gerstenhaber, On the deformation of rings and algebras. III. Ann. of Math. (2) 88 (1968), 1-34.

[16] M. Gerstenhaber, On the deformation of rings and algebras. IV. Ann. of Math. (2) 99 (1974), 257-276.

[17] A. Gohr, On hom-algebras with surjective twisting, J. Algebra 324(2010), 1483-1491.

[18] G. Graziani, A. Makhlouf, C. Menini and F. Panaite, BiHom-associative algebras, BiHom-Lie algebras and BiHom-bialgebras, Symmetry Integrability and Geometry: Methods and Applications, 11(2015), 1-34.

[19] J. T. Hartwig, D. Larsson and S. D. Silvestrov, Deformations of Lie algebras using $\sigma$-derivations, J. Algebra, 295(2016), 314-361.

[20] N. Hu, $q$-Witt algebras, $q$-Lie algebras, $q$-holomorph structure and representations, Algebr. Colloq, 6(1999), 51-70.

[21] F. Kubo, Y. Taniguchi, A controlling cohomology of the deformation theory of Lie triple systems. J. Algebra 278 (2004), 242-250.

[22] L. Liu, A. Makhlouf, C. Menini and F. Panaite, BiHom-pre-Lie algebras, BiHom-Leibniz algebras and Rota-Baxter operators on BiHom-Lie algebras, arxiv: 1706.00474v1(2017).

[23] Y. Ma, L. Chen, J. Lin, One-parameter formal deformations of Hom-Lie Yamaguti algebras, J. Math. Phys. 56(2015), 011701, 12 pp.

[24] A. Makhlouf and S. Silvestrov, Hom-algebra structures, J. Gen. Lie Theory Appl., $2(2008), 51-64$.

[25] A. Makhlouf and S. Silvestrov, Hom-Lie admissible Hom-coalgebras and Hom-Hopf algebras, J. Gen. Lie Theory in Mathematics, Physics and beyond. Springer-Verlag, Berlin, 2009, pp. 189-206.

[26] A. Makhlouf and S. Silvestrov, Hom-algebras and Hom-coalgebras, J. Algebra Appl., 9(2010), 553-589.

[27] J. M. Osborn, Novikov algebras, Nova J. Algebra Geom. 1 (1992), 1-14.

[28] B. Sun, L. Chen, Y. Liu, The construction and deformation of Hom-Novikov superalgebras, arXiv:1501.00229v1(2015).

[29] S. Wang and S. Guo, BiHom-Lie superalgebra structures, arXiv:1610.02290v1 (2016). 
[30] X. Xu, On simple Novikov algebras and their irreducible modules, J. Algebra 185 (1996), 905-934.

[31] L. Yuan, H. You, Hom-Novikov algebras and Hom-Novikov-Poisson algebras. arXiv:1204.6373.

[32] D. Yau, Hom-Novikov algebras, J. Phys. A: Math. Theor. 44 (2011), 085202, 20pp.

[33] D. Yau, A twisted generalization of Novikov-Possion algebras, arXiv:1010.3410v1.

[34] E.I. Zelmanov, On a class of local translation invariant Lie algebras, Sov. Math. Dokl. 35 (1987), 216-218.

[35] R. Zhang, D. Hou, C. Bai. A Hom-version of the affinizations of Balinskii-Novikov and Novikov superalgebras. J. Math. Phys. 52 (2011), 023505, 19 pp. 